\title{
An Evaluation of Malaria Control Practices by Caregivers of Under-5 Children in Calabar South Local Government Area of Cross River State, Nigeria
}

\author{
Agbenin Andrew*, Edem A. Akpan \\ Department of Public Health, Faculty of Allied Medical Sciences, College of Medical Sciences, University of Calabar, \\ Calabar, Cross River State, Nigeria
}

*Corresponding author: Agbenin Andrew; agbenin@yahoo.com

Received 19 June 2019;

Accepted 05 July 2019;

Published 10 July 2019

\begin{abstract}
Background: Despite many years of research and concerted efforts at control of malaria, the achievement of a malaria free world remains a dream. The prevalence of the disease continues to increase in many parts of the world especially among under- 5 children and pregnant women. However, in Nigeria, the scale up of malaria control strategies has produced recognizable public health impact in several communities and this has led to the development of new tools and strategies, including integrated approach to control, aimed at further consolidating malaria control goals. Objectives: To evaluate malaria control practices by Caregivers of under-five children in Calabar South LGA, Cross River State, Nigeria. Methods: A cross sectional study involving the administration of questionnaires to 440 Caregivers of under-five children selected through a multi-stage sampling and simple random sampling techniques. The administration of the questionnaires to the respondents was used to evaluate malaria control practices by Caregivers of under-5 children in Calabar South LGA since the launch of the Roll back Malaria (RBM) Program in Nigeria in 1998. Results: The sociodemographic characteristics of respondents are as follows: $35.3 \%$ of respondents fell within the age brackets of $26-31$ years, $85 \%$ were married, $56.3 \%$ had, at least, secondary school education, $35.8 \%$ were traders, $98.5 \%$ were Christians, $40.8 \%$ were medium income earners, $56.3 \%$ had 3-5 persons per household. Evaluation of Caregivers in the LGA on knowledge of malaria control practices, show they had good knowledge on strategies to control malaria but many of them did not practice them in their homes. The very few that were involved in the practice of malaria control activities rely on vertical approaches to control instead of integrated malaria control efforts as advocated during the launch of the RBM program in Nigeria in 1998. Conclusions: The state Ministry of Health, Calabar, Cross River State, Nigeria, through the Department of Public Health, needs to invest more on education, communication and information dissemination strategies to reach Caregivers of under-5 children to adopt integrated approach to malaria control instead of using isolated vertical approach if they are to achieve the malaria control targets set by RBM and MDGs for under-5 children in Nigeria in general and Calabar South LGA in particular.
\end{abstract}

Keywords: Under-Five Children, Morbidity, malaria control strategies

\section{Introduction}

Malaria is a life-threatening parasitic or protozoan disease caused by infection with parasites of the genus plasmodium and transmitted to man by certain species of infected female Anopheles mosquito. ${ }^{[1,18]}$

Malaria remains the leading cause of child mortality and morbidity in sub-Saharan Africa. The incidence of malaria worldwide is estimated to be 300- 500 million clinical cases each year and death of between 1.1 and 2.7 million people of whom 1.0 million are children under the age of 5 years. Over $90 \%$ of those deaths occur in Africa, and almost $90 \%$ of deaths due to malaria occur in children. ${ }^{[2]}$

Nigeria also has an estimated 57 million cases of malaria a year. Malaria is highly endemic in Nigeria and it remains one of the leading causes of morbidity and mortality in the country with prevalence rate of $919 / 100,000 .^{[2]}$

It has been found that 60 percent of hospital attendance in Nigeria is for malaria treatment, $33 \%$ of young children's death is attributable to malaria (i.e., about 3 out of 10 childhood deaths), twenty-five percent ( 25 percent) of all infant mortality in Nigeria is due to malaria. Every thirty minutes (30minutes), a child dies of malaria in Nigeria. ${ }^{[4]}$

Nigeria, with a population of 198 million people, contributes a quarter of the malaria burden in Africa, i.e., 50 percent of the population will have at least one malaria attack each year. ${ }^{[6,7]}$

High malaria transmission intensity, limited access to adequate treatment, increasing parasite resistance to affordable and safe antimalarial medicines, increasing vectors resistance to widely 
used insecticides, non- use of long-lasting insecticide treated nets (LLINs) in under-5 children and lack of environmental management, delayed care-seeking behavior and inappropriate treatment at home or at community level are factors that contributed to high malaria burden in Africa and in Nigeria in Particular. $^{[8]}$

In the year 2000, following the launch of the Roll Back Malaria (RBM) in Nigeria in 1998, the African Heads of State adopted a variety of strategies to combat the scourge of malaria for the vulnerable population (Under-5 Children and pregnant women). Malaria control, using the RBM approach, follows a horizontal approach and promotes the strengthening of local capacities and health systems so that malaria can be dealt with locally. ${ }^{[1]}$ Following the launch of RBM in Nigeria in 1998, some of the interventions put in place for malaria control in under-5 children include use of ITNs, indoor house spraying, improved malaria treatment including home management of malaria(HMM) and a hypothetical vaccine..$^{[2,3]}$

With the emphasis currently shifting from campaigning to implementation, there is a pressing need for information about the efficacy and cost effectiveness of different intervention strategies, which might guide policy makers on how available resources can be used to obtain the maximum possible social benefit, Knowledge of mosquito bionomics and relevant environmental, social and economic features are extremely important for malaria prevention programs. These programs often consist of personal protection (for examples, the use of repellents, use of ITNs, etc), chemoprophylaxis, etc. ${ }^{[11]}$

To achieve the objective of RBM in reducing malaria-related morbidity and mortality by $25 \%$ in Nigeria by 2005 and by $50 \%$ by 2010 , integrated malaria control practices must be encouraged in Nigeria and particularly among locals living in difficult to reach communities in Nigeria with little or no access to most interventions. ${ }^{[9]}$ In the context of the limited coverage of the population by health facilities in most of the malaria endemic communities in Nigeria, this target seemed quite ambitious and difficult to achieve if integrated malaria control practices are not encouraged. The integrated malaria control approach involves the use of ITNs in under-5 children and other environmental management practices including indoor/outdoor spraying with insecticides and these have been proven to be effective tools for malaria prevention and can significantly reduce severe disease and mortality due to malaria, especially among under-5 children in malaria endemic areas. ${ }^{[12]}$

The scale up of malaria control efforts in recent years, coupled with major investments in malaria research, has produced impressive public health impact in a number of countries and has led to the development of new tools and strategies aimed at further consolidating malaria control goals, which are reduction in transmission and mortality due to malaria especially in under-5 children. ${ }^{[13]}$ The use of ITNs represents a practical means to prevent malaria in Africa in general and in Nigeria in particular, so scaling up coverage to at least $80 \%$ in under-5 children and pregnant women by 2010 is integral to the target set by the Millennium Development Goals(MDGs) and targeting individual protection against malaria in vulnerable groups is an accepted priority. ${ }^{[14]}$ The use of ITNs and house spraying represent a quantum leap on the use of physical barriers and chemicals in malaria control. The use of ITNs alone as a physical barrier provides a remarkable degree of protection against malaria in
Africa. ${ }^{[2]}$ It has been demonstrated in previous research work that both indoor spraying and sleeping under ITN can reduce the prevalence of anemia and the number of malaria-infective mosquitoes biting each night by $90 \%$. ${ }^{[14]}$

In recent times, there has been a strong advocacy for an integrated approach for malaria control. This approach involves the use of effective antimalaria drugs, prompt diagnosis, insecticide sprays, use of ITNs, improved community-based systems (home management of malaria), proper case management, improved health information systems and proper environmental management geared towards vector control. ${ }^{[14,15,17]}$ It was based on this our study seeks to evaluate malaria control practices by Caregivers of under-5 children in Calabar South Local Government Area of Cross River State, Nigeria.

\section{Methods}

\section{Study setting}

The study was conducted in Calabar South LGA of Cross River State with twelve political wards.

According to the provisional results of the Federal Republic of Nigeria 2006 population census, Calabar South LGA has a total population of 191,515 people made up of 94,584 males and 96,931 females (National Population Census, 2006). It has a 4-year population growth rate of about $8.5 \%$ giving a projected total population of about 240,350 people by year 2019 (National Population and Housing Census, 2006).

Calabar South LGA is bounded on the North by Calabar Municipality Local Government Area, on the South by the Atlantic Ocean, on the East by Akpabuyo Local Government Area, and on the West by Odukpani Local Government Area. This area lies within the tropical rain forest belt of Southeastern Nigeria. It has an annual rainfall of over $3500 \mathrm{~mm}$, with the rainy season lasting from April-November. ${ }^{[18]}$

The predominant ethnic group is Efiks. The people's main occupations are subsistence farming, fishing and logging. Malaria is holoendemic in this study area with high and perennial transmission especially in the rainy season (from April November). Calabar South LGA is lying on $4^{0} 45^{1}$ and $4^{0} 58^{1}$ North latitudes, $8^{0} 15^{\mathrm{I}} \mathrm{E}$ and $8^{0} 25^{\mathrm{I}} \mathrm{E}$ longitudes. ${ }^{[18]}$

\section{Study Design}

This study was a cross-sectional study designed to evaluate malaria control practices by Caregivers of under-5 children in Calabar South LGA of Cross River State, Nigeria

\section{Target population}

The target population for this study was Caregivers of under-5 Children and the inclusion criteria were Caregivers who are women of reproductive age and such women must have lived in Calabar South LGA for a minimum of one year and above

\section{Sample size Determination}

The sample size for this study was determined by using the Lutz's formula of 1982. The formula states that:

$$
n=Z^{2} \alpha \frac{p q}{e^{2}}
$$

Using this formula, a sample size of 400 with a $10 \%$ consideration for attrition gave a final sample size of approximately 440 that 
were administered with the questionnaire from which responses from 400 respondents were used for the study

Using this formula to calculate the sample size that was used for the study was more appropriate because it is a formula for calculating sample size of dichotomous descriptive study or prevalence study where:

$\mathrm{n}=$ the desired sample size

$\mathrm{Z}_{\alpha}=$ the alpha level of the confidence interval (in this case $95 \%=1.96=2$ : this value is the standardized normal distribution corresponding to stated confidence level of $95 \%$ )

$\mathrm{P}=$ proportion of occurrence

$q=(1-p)$ proportion of non-occurrence

$\mathrm{e}=$ precision required $(5 \%)$

$\mathrm{n}=$ the desired sample size

\section{Method of Sampling}

In this study, a combination of multi-stage sampling and simple random sampling techniques was used to select the final respondents of 440 caregivers.

\section{Data Management/Instrument for Data Collection}

This involved the administration of either self-administered or researcher-administered structured questionnaires on 440 respondents following seeking their informed consents to participate in the study. Following data cleaning and validation, the data from 400 respondents (Caregivers of under-5 children) were the ones that were eventually used for analysis in the study taking into consideration of some respondents who dropped out from the study and the voiding of incompletely filled questionnaires by some respondents

The questionnaire used for the study was prepared in four sections. The first section was an introductory section of what the study was all about and information on informed consent, the second section was to collect the socio-demographic characteristics of the respondents, the third section was to collect information on their knowledge of malaria control strategies and other knowledge in malaria bionomics and the fourth section was designed to collect information on their level of malaria control practices at their various homes looking specifically for information on integrated malaria control practices

Data entry and analysis were done using the Statistical Package for Social Scientist (SPSS) version 21.

The data types analyzed using this quantitative statistical analysis program involved data for assessment of Caregivers of under-5 children's knowledge on the different malaria control strategies they knew, especially in vulnerable groups, what their experiences at home on malaria control practices in vulnerable groups were. All these were reported and summarized using descriptive statistics.

To establish whether there was a relationship between Caregivers' income based, on the national minimum wage of N18,000/month, and its influence on their under-5 children sleeping under ITN the previous night, inferential statistical analysis using Chisquared $\left(\mathrm{X}^{2}\right)$ determined at $5 \%$ level of significance $(\mathrm{P}$ Value $<0.05)$ was used for the analysis.

\section{Ethical Consideration}

Ethical clearance and approval for the study protocol was reviewed and approved by the Cross River State Health Research Ethics Committee of the Cross River State Ministry of Health

\section{Results}

The result of socio-demographic characteristics of respondents used for the study as seen in Table 1 below shows that a total of $141(35.3 \%)$ respondents, which constitute the highest number of respondents, fall within the age range of $26-31$ years, $340(85 \%)$ of the respondents, which constitute the highest number of respondents, are married,225 (56.3\%) of respondents, representing the highest number of respondents, had at least secondary school education, 143(35.8\%) of the respondents, constituting the highest number of respondents, owned a private business,394(98.5\%) of the respondents, which represent the highest number of respondents, are Christians,163 (40.8\%) of the respondents, representing the highest number of respondents, have an income that falls within the country monthly minimum wage of N18,000 naira and $225(56.3 \%)$ respondents, representing the highest number of respondents, have between 3-5 persons per household.

Table 1: Socio-demographic variables of respondents

\begin{tabular}{|c|c|c|}
\hline Variable & Frequency & Percentage (\%) \\
\hline \multicolumn{3}{|l|}{ Age group (years) } \\
\hline $14-19$ & 13 & 3.3 \\
\hline $20-25$ & 137 & 34.3 \\
\hline $26-31$ & 141 & 35.3 \\
\hline $32-37$ & 66 & 16.5 \\
\hline $38-43$ & 29 & 7.3 \\
\hline 44 and above & 14 & 3.5 \\
\hline Total & 400 & 100.0 \\
\hline \multicolumn{3}{|l|}{ Marital status } \\
\hline Divorced & 1 & 0.3 \\
\hline Married & 340 & 85 \\
\hline Separated & 2 & 0.5 \\
\hline Single & 48 & 12 \\
\hline Widow & 9 & 2.3 \\
\hline Total & 400 & 100 \\
\hline \multicolumn{3}{|l|}{ Educational status } \\
\hline No formal education & 19 & 4.80 \\
\hline Primary & 43 & 10.8 \\
\hline Secondary & 225 & 56.3 \\
\hline Tertiary & 113 & 28.3 \\
\hline Total & 400 & 100.0 \\
\hline \multicolumn{3}{|l|}{ Occupation } \\
\hline Artisan & 80 & 20.0 \\
\hline Civil servant & 46 & 11.5 \\
\hline Farming & 1 & 0.30 \\
\hline Student & 21 & 5.30 \\
\hline Trading & 143 & 35.8 \\
\hline Others (no work) & 108 & 27.0 \\
\hline Non-response & 1 & 0.30 \\
\hline Total & 400 & 100.0 \\
\hline \multicolumn{3}{|l|}{ Religion } \\
\hline Christianity & 394 & 98.5 \\
\hline Islam & 5 & 1.30 \\
\hline Others & 1 & 0.30 \\
\hline Total & 400 & 100.00 \\
\hline $\begin{array}{l}\text { Income per month (based } \\
\text { on national minimum wage } \\
\text { of 2012) }\end{array}$ & Frequency & Percentage (\%) \\
\hline High income & 51 & 12.8 \\
\hline Medium income & 163 & 40.8 \\
\hline Low income & 43 & 10.8 \\
\hline
\end{tabular}




\begin{tabular}{|l|l|l|}
\hline No reliable source of income & 140 & 35.0 \\
\hline Non-response & 3 & 0.75 \\
\hline Total & 400 & 100.00 \\
\hline $\begin{array}{l}\text { Number of persons per } \\
\text { household }\end{array}$ & Frequency & Percentage (\%) \\
\hline $1-2$ & 17 & 4.30 \\
\hline $3-5$ & 225 & 56.3 \\
\hline $6-8$ & 128 & 32.0 \\
\hline Greater or equal to 9 & 29 & 7.30 \\
\hline Non-response & 1 & 0.30 \\
\hline Total & 400 & 100.00 \\
\hline
\end{tabular}

On study of the knowledge of Caregivers on malaria control practices that constitute the national strategies adopted during the launch of RBM in the year 1998 to reduce malaria morbidity and mortality in under-5 children, Table 2 below shows that 400(100\%) of Caregivers have knowledge on health facility-based case management of malaria in under-5 children. On assessment of Caregivers' knowledge on Home Management of Malaria (HMM) in under-5 children, only $120(30 \%)$ seem to have knowledge on HMM in under-5 children while $280(70 \%$ ) do not have knowledge, On malaria prevention activities in under-5 children, 400(100\%) Caregivers of under-5 children are knowledgeable on indoor and outdoor spraying as strategy to reduce the transmission of malaria, their subsequent knowledge in other environmental practices as well as the use of ITNs to prevent malaria transmission is equally appreciable as about (347)87\% and (397)99\% respectively of Caregivers have knowledge of these practices. Further study also shows in Table 2 below that Caregivers of under- 5 children have knowledge of where to source ITNs for home use in the control of malaria in under-5 children.

Table 2: Knowledge of malaria control activities by Caregivers of under-5 children in Calabar South LGA, Calabar, Cross River State

\begin{tabular}{|c|l|l|l|l|}
\hline S/N & Description & Have Knowledge (\%) & No knowledge (\%) & Total (Total \%) \\
\hline 1 & Home management of malaria in under-5 children & $120(30)$ & $280(70)$ & $400(100)$ \\
\hline 2 & $\begin{array}{l}\text { Facility based management of malaria in under-5 children } \\
\text { (Tertiary hospital, Secondary Hospital, PHC) }\end{array}$ & $400(100)$ & $0(0)$ & $400(100)$ \\
\hline 3 & Under-5 children sleeping under ITNs & $397(99.3)$ & $3(0.7)$ & $400(100)$ \\
\hline 4 & Indoor/outdoor spraying with insecticide & $400(100)$ & $0(0)$ & $400(100)$ \\
\hline 5 & $\begin{array}{l}\text { Environmental management (Bush clearing, discarding } \\
\text { stagnant water near homes, etc) }\end{array}$ & $347(87)$ & $53(13)$ & $400(100)$ \\
\hline 6 & Where to source your ITNs & $397(99.3)$ & $3(0.7)$ & $400(100)$ \\
\hline
\end{tabular}

Further inquisition to evaluate the extent of malaria case management practices by Caregivers of under-5 children of Calabar South LGA that could contribute significantly to reduction of malaria morbidity and mortality in under-5 children resident in the LGA, Table 3 below shows that 216(54\%) of Caregivers of under-5 children practice HMM in under-5 children while $170(43 \%)$ practice facility-based care for their under-5 children with malaria.

On malaria control practices by caregivers of under-5 children in Calabar South LGA of Cross River State, Table 3 also shows that $216(54 \%)$ of Caregivers of under-5 children practice HMM of malaria while $170(43 \%$ ) choose facility-based treatment option for their children under-5 with malaria infection.For non-treatment malaria control practices by Caregivers of under- 5 children in Calabar South LGA,398 (99.5\%) of Caregivers, representing the highest number of respondents, engage in environmental management (bush clearing around their houses/disposal of stagnant water) as preventive measures for malaria transmission. For respondents whose child or children slept under ITN the previous night, the result from Table 3 shows that only 126(32\%) of respondents affirmed that their under- 5 children slept under ITN the previous night while respondents who indulge in indoor and outdoor spraying to kill malaria parasites-carrying vectors was only $23(6 \%)$.

Table 3: Malaria control practices by caregivers of under-5 children in Calabar South LGA of Cross River State, Nigeria

\begin{tabular}{|l|l|l|l|}
\hline Description & Practice (\%) & Do not Practice (\%) & Total (Total \%) \\
\hline Home management of malaria in under-5 children & $216(54)$ & $184(46)$ & $400(100)$ \\
\hline Facility-based treatment of under-5 children with malaria & $170(43)$ & $230(57)$ & $400(100)$ \\
\hline Environmental management (bush clearing/disposal of stagnant water) & $398(99.5)$ & $2(0.5)$ & $400(100)$ \\
\hline Under-5 children who slept under ITN last night & $126(32)$ & $274(68)$ & $400(100)$ \\
\hline Indoor/outdoor spraying & $23(6)$ & $377(94)$ & $400(100)$ \\
\hline $\begin{array}{l}\text { Others (not listed as one of the malaria control strategies during RBM } \\
\text { Launch) }\end{array}$ & $14(4)$ & $386(96)$ & $400(100)$ \\
\hline
\end{tabular}

On relationship between income of Caregivers of under-5 children and its influence on their under-5 children sleeping under ITNs the previous night, the result on Table 4 below shows this is not significant (Chi-square $=3.9085 \mathrm{df}=3 \mathrm{P}=0.2715$ ) as ITNs are hugely supported by Donor-funded Organizations and for this reason they are usually distributed to Primary Healthcare Centers(PHCs) in Nigeria and in this LGA free of charge for residents to have easy access to this intervention.

Table 4: Relationship between income and child sleeping under ITN the previous night

\begin{tabular}{|l|c|c|c|}
\hline \multirow{2}{*}{ Income Level } & Child Slept under ITN the previous night & \multirow{2}{*}{ Total } \\
\cline { 2 - 4 } & Yes & 32 & 51 \\
\hline High Income Earners & 19 & 118 & 163 \\
\hline Medium Income Earners & 45 & 33 & 50 \\
\hline Low Income Earners & 17 & 91 & 136 \\
\hline No reliable source of income & 45 & 274 & 400 \\
\hline Total & 126 & & \\
\hline
\end{tabular}


On the choice of antimalarial drugs used by Caregivers of under-5 children for HMM in under- 5 children, Figure 1 below shows that the most common antimalarial medicines used by Caregivers of under-5 children for HMM in under-5 children in the LGA was artemisinin-based combination therapies (ACTs) and this represented $58.2 \%$ of commonly used antimalarial drugs by Caregivers of under-5 children for HMM of malaria in Calabar South LGA, of Cross River State, Nigeria.

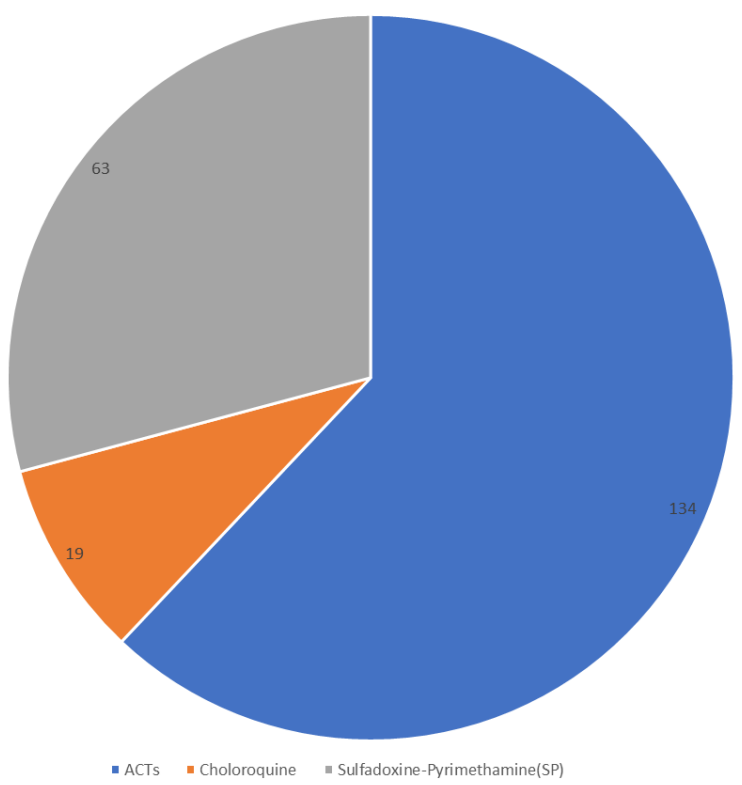

Figure 1: Antimalarial drugs used by caregivers of under - 5 children in HMM

\section{Discussions}

On study of knowledge of malaria control practices as adopted as national strategies to roll back malaria to impact on reduction in malaria cases and deaths in under-5 children, ${ }^{[1,2,6]}$ the result from Table 2 shows that Caregivers of under- 5 children have very good knowledge on strategies to roll back malaria, however, their good knowledge on malaria control does not translate into practice of these activities to curb malaria infection especially in the vulnerable groups of Under-5 children and pregnant women in the area. From the results presented on Table 3, (216)54\% of Caregivers of under-5 children do practice HMM in under-5 children and this still falls short of the 2005 RBM target, called the Abuja target, of ensuring $60 \%$ of under- 5 children with malaria are treated presumptively with antimalarial drugs at home within 24 hours of detection of fever. ${ }^{[2,7,9]}$ The results in Table 3 equally show that only (126)32\% of Caregivers of under-5 children slept under ITNs every night while a mere (23)6\% of them use indoor/outdoor spraying as strategies to control malaria in this community. On environmental management that involves bush clearing and disposal of stagnant dirty water around homes, about (398) $99.5 \%$ of respondents do agree to the practice of this as a way of preventing transmission of malaria in this community. From the analysis of these results, it is obvious the residents of this community do not practice integrated approach in the control of malaria infection and transmission, but rather vertical approaches are used in malaria control. Such practices will make it difficult to achieve the MDGs and RBM Program targets of ITN use coverage by over $80 \%$ by year 2010 and reduction by $25 \%$ of malaria morbidity respectively in Sub-Saharan African Countries with holo-endemic transmission of malaria. Integrated malaria control practices remain the only viable option at reducing mortality and morbidity ${ }^{[2,9]}$. Previous research has demonstrated that a combined use of ITNs and indoor spraying with insecticides as one of the strategies for malaria control has the capacity to reduce the ability of malaria-carrying mosquitoes to bite its victims by $90 \%$ every night ${ }^{[14]}$ but yet from this study only $32 \%$ and $6 \%$ respectively of Caregivers are involved in these practices as strategies to control malaria in this community.

From the analysis of the type of antimalarial commonly used by Caregivers for HMM practice in under-5 children, the result from Figure 1 shows that from the 54\% of Caregivers that practice HMM in under-5 children, $62 \%$ of them use the artemisinin-based combination therapies (ACTs) as their first line antimalarial drugs of choice and this is in line with WHO and Nigerian national malarial treatment recommendation guidelines for management of malaria in under-5 children. ${ }^{[2,6,8,10]}$

On further study to establish the relationship between income status of Caregivers of under-5 children and use of ITNs, result from Table 4 shows this is not significant (Chi-square $=3.9085$, $\mathrm{df}=3, \mathrm{P}=0.2715$ ), this could probably be attributable to ITNs being distributed to PHCs and homes in these communities free of charge because of Donor-funded Agencies support to free distribution of ITNs to intended users.

The recommendation by WHO and RBM Program is that for effective malaria control in high transmission areas, and especially in vulnerable population sub-groups of under-5 children and pregnant women, an integrated malaria control approach is best way to reduce malaria burden and malaria-related mortality if we are to meet the RBM and MDGs targets for malaria control. ${ }^{[14,16,17]}$

\section{Limitations of the Study}

One limitation of the study is recall bias by Caregivers of under-5 children in response to some of the questions asked by the research team but this could not in any way invalidate the findings from this study

\section{Conclusions}

Despite several years of research and concerted efforts at malaria control, the realization of a malaria-free world remains a mirage. However, the scale up of malaria control activities and plans in recent years has resulted in impressive public health impact in Nigeria and has led to the development of new tools and control strategies aimed at further consolidating malaria control goals. Despite the milestones the Caregivers of under-5 children have recorded in some aspects of malaria control activities in under-5 children in Calabar South LGA of Cross River State, there is still need by the State Ministry of Health, through the Department of Public Health, to re-emphasize the need by residents of Calabar South LGA, Calabar, Cross River State, Nigeria in particular and residents of Cross River State in general to embrace and practice integrated malaria control approaches to roll back malaria in under5 children through carefully organized information dissemination to Caregivers of under-5 children on the benefits of using integrated preventive practices at control. Applying this strategy, follows the RBM approach that promotes the strengthening of local capacities and health systems so that malaria can be dealt with locally with the overall aim of contributing to the overall reduction in malaria cases and deaths in Calabar South LGA of Cross River state,Nigeria 


\section{Acknowledgements}

We wish to thank Dr. Abraham Amlogu, of the Department of Pharmacy, State House Medical Center, Aso Rock, Abuja for thoroughly critiquing the manuscript and for making useful suggestions that helped to improve its quality.

\section{References}

[1] Naboro D and Mendis K (2000). Roll Back Malaria is unarguably both necessary and possible. Bulletin of the World Health Organization 78,1454-1455

[2] World Health Organization reports on global strategy for malaria control, Geneva 2008

[3] Goodman CA and Mills AJ (1999). Evidence base on the cost-effectiveness of malaria control measures in Africa. Health Policy and Planning 14,301-312

[4] Oresanya DB, Moshe H, Sofola OT (2008). Utilization of insecticide-treated nets (ITNs) by Under- 5 children in Nigeria. Malaria journal 7:145.

[5] World Health Organization report 2002.Reducing risks, promoting health, Geneva 2002.

[6] Federal Ministry of Health. National malaria control program/FMOH/2005. Annual Report 2005.

[7] Edugie A, Mosanya ME, Chioma A, Olapeju O, Ezedinachi ENU, Afolabi BM, Bayo F, Gemade EI, Yomi O, Akinpelumi AA, Jackson S, James B, Prudence S, Ifeanyi I, Yeboah-Antwik K, Aaron KO. Nigeria roll back malaria country consultative mission final report: essential actions to Support the attainment of the Abuja targets, 2004.

[8] Sirima SB, Konate A, Tiono AB, Convelbo N, Cousens S, Pagnoni F (2008). Implementation of Home-based management of malaria in children reduces the workload for peripheral health Facilities in a rural district of Burkina Faso. Malaria journal 7:201.
[9] World Health Organization. The African summit on roll back malaria, Abuja, Nigeria, April 25, 2000, Geneva (Document WHO/CDS/RBM/200.17)

[10] Sirima SB, Konate A, Tiono AB, Convelbo N, Cousens S, Pagnoni F (2003). Early treatment of Childhood fevers with pre-packaged antimalarial drugs in the home reduces severe malaria Morbidity in Burkina Faso. Trop Med Int Health 8:133-139.

[11] Hutubessy RCW, Bendib LM and Evans DB (2001). Critical issues in the economic evaluation of interventions against communicable diseases. Acta Tropica 78,191-206.

[12] Yazoume YE, et al, (2012). Can universal ITN campaigns achieve equity in Coverage and use? The case of northern Nigeria. Malaria Journal 11:32

[13] Bianca JD and Robert DN (2012). Strengthening the policy setting process for global malaria control and elimination. Malaria Journal 11:28

[14] Curtis CF (1999). Malaria control: bed nets or spraying? Background and Trial in Tanzania. Unpublished Roy. Soc. Trop. Med. Hyg Meeting on bed nets.

[15] Daniel MNO (1999). An integrated approach for malaria control in Africa. Malaria and infectious diseases in Africa 10:4-13.

[16] WHO (2002). RBM;2001-2010: United Nations Decade to Roll Back Malaria. Geneva.

[17] Daniel MNO (1999). An integrated approach for malaria control in Africa. Malaria and Infectious Diseases in Africa 10:4-13.

[18] Bernice U, Aniekan P, Sony-Shakins U, Alaribe A (2018). Prevalence of malaria parasites amongst pregnant women in Calabar, Cross River State, Nigeria. International journal of healthcare and medical sciences, vol.4, Issue 11, PP:193-195. 